\title{
Compulsive-Like Sufentanil Vapor Self-Administration in Rats
}

\author{
Janaina CM Vendruscolo', Brendan J Tunstall', Stephanie A Carmack', Brooke E Schmeichel', \\ Emily G Lowery-Gionta', Maury Cole ${ }^{2}$, Olivier George ${ }^{3}$, Sophia A Vandewater ${ }^{3}$, Michael A Taffe ${ }^{3}$, \\ George F Koob' and Leandro F Vendruscolo*,I
}

'Intramural Research Program, National Institute on Drug Abuse, National Institutes of Health, Baltimore, MD, USA; ${ }^{2}$ La Jolla Alcohol Research, La Jolla, CA, USA; ${ }^{3}$ Department of Neuroscience, The Scripps Research Institute, La Jolla, CA, USA

\begin{abstract}
Opioid misuse is at historically high levels in the United States, with inhalation (ie, smoking and vaping) being one of the most common routes of consumption. We developed and validated a novel preclinical model of opioid self-administration by inhalation that does not require surgery and reliably produces somatic and motivational signs of dependence. Rats were trained to perform an operant response (nosepoke) to receive $10 \mathrm{~s}$ of vaporized sufentanil, a potent opioid, in $2 \mathrm{~h}$ daily sessions. Rats readily and concentration-dependently self-administered vaporized sufentanil. Rats exhibited a significant increase in responding for sufentanil when given the preferential $\mu$-opioid receptor inverse agonist naloxone, suggesting the participation of $\mu$-opioid receptors in the reinforcing properties of sufentanil vapor. Serum sufentanil concentrations significantly correlated with the number of sufentanil vapor deliveries. Rats that were given long access (LgA; 12 h/day) but not short access (ShA; I h/day) to vaporized sufentanil escalated their drug intake over time and exhibited both naloxone-precipitated somatic signs of opioid withdrawal and spontaneous withdrawal-induced mechanical hypersensitivity. After 6 months of forced drug abstinence, LgA rats returned to pre-escalation baseline levels of responding for sufentanil and mechanical sensitivity. Upon subsequent re-escalation (ie, after the retum to extended access to sufentanil vapor), LgA rats again developed naloxone-precipitated somatic signs of withdrawal and spontaneous withdrawal-induced mechanical hypersensitivity. These findings demonstrate that the operant sufentanil vapor self-administration model has both face and construct validity and therefore will be useful for investigating the neurobiological basis of opioid addiction. Neuropsychopharmacology (2018) 43, 80 I-809; doi:I0.1038/npp.2017.172; published online 13 September 2017
\end{abstract}

\section{INTRODUCTION}

Opioid misuse and overdose in the United States have reached the highest rates in recorded history. This major public health crisis is driven by both a steady increase in the misuse of prescription opioids (eg, pain relievers) and a recent surge in heroin abuse (Dowell et al, 2016; Wilkerson et al, 2016). Because of its relatively low production cost and high potency, fentanyl (30-50 times more potent than heroin) and its analog sufentanil (5-10 times more potent than fentanyl) are common adulterants in heroin and have been hypothesized to contribute significantly to deaths that are caused by opioid overdose (Frank and Pollack, 2017). Although evidence indicates that fentanyl and sufentanil are the most abused opioids among anesthesiologists (Kintz et al, 2005), the contribution of fentanyl and its analogs to opioid-related deaths in the general population is less known, partially because these drugs are still frequently omitted from toxicology tests (Centers for Disease Control and Prevention, 2008).

\footnotetext{
* Correspondence: Dr LF Vendruscolo, Neurobiology of Addiction Section, NIH/NIDA - IRP/INRB, 25I Bayview Boulevard, BRC Room 08A727, Baltimore, MD 21224, USA, Tel: + I 443740 2869, Fax: + I 443740 2827, E-mail: leandro.vendruscolo@nih.gov

Received 18 June 2017; revised 18 July 2017; accepted 26 July 2017; accepted article preview online 16 August 2017
}

Users primarily initiate the use of opioids orally and by inhalation (ie, smoking and vaping), whereas experienced users consume opioids most frequently by inhalation and intravenous injection (for a review, see Gasior et al, 2016). When opioids are self-administered intravenously or by inhalation, they produce rapid and potent euphorigenic effects that contribute to their abuse liability. The intravenous selfadministration of opioids (eg, heroin, morphine, oxycodone, buprenorphine, and fentanyl) is the most commonly used procedure in preclinical models of addiction. By varying the period of opioid access, intravenous opioid self-administration can be used to model the transition from controlled drug use to compulsive-like drug seeking. Rats that are allowed long access (LgA, 6-23 h per day) to intravenous opioids escalate their drug intake over time and exhibit greater levels of drug seeking (Ahmed et al, 2000; Barbier et al, 2013; Lenoir and Ahmed, 2007, 2008; Vendruscolo et al, 2011; Wade et al, 2015; Schmeichel et al, 2015), hypohedonia (Kenny et al, 2006), allodynia (Barbier et al, 2013; Edwards et al, 2012; Park et al, 2015), anxiety-like behavior (Park et al, 2013), and somatic signs of opioid withdrawal (Vendruscolo et al, 2011) compared with rats that are allowed limited or short access (ShA; $1-3 \mathrm{~h}$ per day) to the drug. Moreover, a few studies have shown that rats with a history of escalated heroin selfadministration started at escalated responding levels and further escalated when given access to heroin again after 
2 weeks (Wade et al, 2017) or 1 month (Schlosburg et al, 2013) of forced drug abstinence.

In contrast to intravenous opioid self-administration, few preclinical studies have used self-administration via inhalation. This is possibly because of the lack of well-validated apparatus for typical laboratory species, such as rats or mice. Previous studies used a self-constructed nebulizer-based system and found that rats performed a dose-dependent operant response for sufentanil vapor (Jaffe et al, 1989; Weinhold et al, 1993). It is not currently known whether opioid self-administration by inhalation produces an escalation of drug intake and somatic and motivational signs of opioid dependence. Also unknown is whether the signs of opioid dependence normalize after a long period of drug abstinence.

Because of the paucity of animal models that use inhalation as the route of drug self-administration, we sought to develop a surgery-free model of opioid addiction using operant self-administration of vaporized sufentanil that was intended to model smoking and vaping in humans. The delivery system was based on e-cigarette technology to reflect the broadening availability of such products, which are easily adapted for delivery of psychoactive non-nicotine substances. We found that rats self-administered sufentanil for its pharmacological effects in amounts that led to detectable serum opioid levels. We also found that $\operatorname{LgA}$ rats but not ShA rats escalated sufentanil vapor selfadministration over time and exhibited somatic and motivational signs of opioid dependence. Finally, using a longitudinal approach, we demonstrated that the behavior of rats (responding for sufentanil vapor and mechanical sensitivity) with a history of opioid dependence returned to baseline (BL; pre-escalation) levels after long-term drug abstinence but readily re-escalated with renewed access.

\section{MATERIALS AND METHODS}

\section{Subjects}

Thirty-three adult male Wistar rats were obtained from Charles River (Kingston, New York, NY, USA). The rats weighed $250-275 \mathrm{~g}$ at the beginning of the study. The rats were group-housed (two per cage) and maintained under a reverse $12 \mathrm{~h} / 12 \mathrm{~h}$ light/dark cycle (lights off at 0800 hours and on at 2000 hours) at $21^{\circ} \mathrm{C} \pm 2{ }^{\circ} \mathrm{C}$. The animals had free access to food and water throughout the study, except as noted below. All procedures were conducted per the National Institutes of Health Guide for the Care and Use of Laboratory Animals and were approved by the National Institute on Drug Abuse, Intramural Research Program, Animal Care and Use Committee.

\section{Apparatus}

Operant vapor self-administration was conducted in eight air-tight chambers that measured $26 \times 26 \times 38 \mathrm{~cm}$ (Allentown, Allentown, NJ, USA). The chambers were housed in a dimly lit room inside a dark Plexiglas enclosure that minimized light and external noise. Two nosepoke holes $(2.5 \mathrm{~cm}$ diameter) were mounted $4.5 \mathrm{~cm}$ above the floor on the back wall of the chamber. White light bulbs were mounted $6 \mathrm{~cm}$ above the floor on the back wall of the chamber to the outer side of each nosepoke hole. Chamber airflow was vacuum controlled by an exhaust pump that pumped ambient air at $1 \mathrm{liter} / \mathrm{min}$. The air outlet was located at the top of the back wall of the chamber $(23 \mathrm{~cm}$ above the floor) and connected through tubing to a large activated charcoal filter (Carbtrol Corporation G-0.5, Bridgeport, CT, USA). This suction system allowed the flow of vaporized drug into the chamber when the drug delivery system was activated. The vapor port was located on the left front side of the chamber, $7 \mathrm{~cm}$ above the floor (ie, the opposite corner from the air outlet). The drug solution was contained in a glass tank (Protank 3) that was equipped with an atomizer (Kanger Tech, Shenzhen Kanger Technology, Fuyong Town, Shenzhen, China) that was activated by a custom e-cigarette device (La Jolla Alcohol Research, La Jolla, CA, USA). Med Associates software and an interface (St Albans, VT, USA) recorded nosepokes and controlled activation of the electronic vaporizer, presentation of the cue light, and operation of the exhaust valve (ON/OFF). Supplementary Video S1 shows a rat performing a nosepoke for access to sufentanil vapor in the operant chamber.

\section{Drugs}

Sufentanil citrate (National Institute on Drug Abuse, Intramural Research Program Pharmacy, Baltimore, MD, USA), a highly potent synthetic opioid, was added to an 80/20 mixture of propylene glycol/vegetable glycerol. Nosepokes in the active hole (left side of the back wall) resulted in $10 \mathrm{~s}$ delivery of vaporized sufentanil $(1.65-10 \mathrm{mg} / \mathrm{ml})$ through a vapor entrance port located on the left front side of the chamber and initiation of a $60 \mathrm{~s}$ timeout period that was signaled by a cue light, during which the exhaust valve was shut OFF (ie, drug vapor that spreads to the chamber was not evacuated during the timeout period). After the timeout period, the exhaust valve was activated again, and any remaining vapor was quickly removed from the chamber. Nosepokes in the inactive hole (right side of the back panel) were recorded throughout the entire session (including the $60 \mathrm{~s}$ timeout period initiated by nosepoke of the active hole) as an indicator of general activity but had no programmed consequences (ie, it did not initiate a $60 \mathrm{~s}$ timeout period). Naloxone $(0.12-1 \mathrm{mg} / \mathrm{kg}$; Mylan Institutional, Galway, Ireland) was dissolved in saline and injected subcutaneously in a volume of $1 \mathrm{ml} / \mathrm{kg}$.

\section{Concentration-Response Function for Sufentanil Vapor Self-Administration}

All behavioral tests were conducted during the dark phase of the light/dark cycle, 3-5 days per week. No food or water restriction was used to establish operant responding at any point of training. The rats did not have access to food or water while they were tested in ShA sessions $(1-2 \mathrm{~h})$. The rats $(n=8)$ were trained to nosepoke on a fixed-ratio 1 (FR1) schedule of reinforcement (ie, each operant response in the active hole resulted in drug delivery) to obtain $10 \mathrm{~s}$ of sufentanil vapor $(3.33 \mathrm{mg} / \mathrm{ml})$ delivery and $60 \mathrm{~s}$ vapor evacuation shut off in $2 \mathrm{~h}$ sessions for six sessions. This initial training dose was determined in pilot studies to achieve approximately five drug deliveries per hour, which is comparable to the number of infusions per hour that was obtained in our previous studies using intravenous heroin 
$(60 \mu \mathrm{g} / \mathrm{kg} /$ infusion) self-administration (Vendruscolo et al, 2011). The rats were then given the opportunity to selfadminister $1.65 \mathrm{mg} / \mathrm{ml}$ sufentanil for eight sessions and $10 \mathrm{mg} / \mathrm{ml}$ for four subsequent sessions. The data are expressed as the number of reinforcers (ie, drug vapor deliveries) that were obtained in $2 \mathrm{~h}$.

\section{Effect of Naloxone on Sufentanil Vapor Self- Administration}

The same rats $(n=8)$ that were used in the drug concentration-response function experiment (described above) were used for this experiment. The rats were subcutaneously injected with ascending doses of naloxone $(0.12,0.25,0.5$, and $1 \mathrm{mg} / \mathrm{kg}$ ) immediately prior to $2 \mathrm{~h}$ self-administration sessions with FR1 access to $10 \mathrm{mg} / \mathrm{ml}$ sufentanil. Baseline sessions (ie, without naloxone injection) of sufentanil vapor self-administration were alternated with naloxone testing sessions. The data from five $\mathrm{BL}$ sessions were averaged to compare with the effects of different doses of naloxone. The data are expressed as the number of reinforcers (ie, drug vapor deliveries) that were obtained in $2 \mathrm{~h}$ at varying time points $(30,60,90$, and $120 \mathrm{~min})$.

\section{Evaluation of Serum Sufentanil Concentrations}

Serum (blood) sufentanil concentrations were measured in a separate cohort of rats $(n=9)$ following passive drug exposure. The rats received 2,5 , or 10 non-contingent, $10 \mathrm{~s}$ sufentanil vapor $(3 \mathrm{mg} / \mathrm{ml})$ deliveries $(n=3$ rats per condition) in a $2 \mathrm{~h}$ period. The onset of vapor deliveries across the $2 \mathrm{~h}$ period was evenly spaced and timed so that the final vapor delivery occurred at minute 118 of the session, regardless of the number of deliveries (see Figure 2a). At the end of the session, the rats were removed from the chambers and deeply anesthetized with isoflurane. A blood sample $(6 \mathrm{ml})$ was collected from each rat via direct cardiac puncture and centrifuged at 4000 rotations per minute for $10 \mathrm{~min}$ at $4^{\circ} \mathrm{C}$. Serum $(3 \mathrm{ml})$ was transferred to plastic tubes and frozen at $-20^{\circ} \mathrm{C}$. Plasma sufentanil concentrations were analyzed by high-performance liquid chromatography/tandem mass spectrometry at NMS Labs (Willow Grove, PA, USA). The data are expressed as $\mathrm{ng} / \mathrm{ml}$ of serum.

\section{Escalation and Re-Escalation of Sufentanil Vapor Self- Administration}

A separate cohort of rats $(n=16)$ was used to assess the escalation and re-escalation (after 6 months of forced abstinence) of sufentanil vapor self-administration. Mechanical sensitivity (von Frey test, described below) and naloxone-precipitated withdrawal (described below) were assessed during both escalation and re-escalation phases.

Based on the concentration-response function experiment described above, we chose $3 \mathrm{mg} / \mathrm{ml}$ of sufentanil as the training dose. The rats were given the opportunity to selfadminister sufentanil on an FR1 schedule in $1 \mathrm{~h}$ sessions for eight sessions. They were then divided into two groups that were matched for operant responding: ShA (1 h of sufentanil access/day; $n=8)$ and $\operatorname{LgA}(12 \mathrm{~h}$ of sufentanil access/day; $n=8)$. Both groups underwent 13 vapor self-administration sessions. The $\operatorname{LgA}$ rats had free access to food and water in the self-administration chambers, along with access to 'chew toys' (unflavored Nylabones) to minimize self-directed stereotyped behavior. The same procedure was repeated after 6 months of forced drug abstinence to assess the reescalation of sufentanil vapor self-administration. The data are expressed as the number of reinforcers earned in 1 and $12 \mathrm{~h}$.

\section{Measurement of Mechanical Sensitivity}

Mechanical sensitivity was evaluated using von Frey filaments as previously reported (Edwards et al, 2012; Park et al, 2015). Testing occurred before drug exposure (BL) and just prior to session 2 (Time 1; T1) and session 10 (Time 2; T2) of the escalation test described above. The rats were acclimated for $15 \mathrm{~min}$ in elevated cages with a wire mesh floor. A series of von Frey filaments were applied perpendicularly to the plantar surface of the hind paw. Withdrawal of the hind paw indicated a positive response. The stimulus was incrementally increased until a positive response was observed and then decreased until a negative response was observed to determine the paw withdrawal threshold as previously reported (Chaplan et al, 1994; Edwards et al, 2012). The data are expressed as grams of applied force.

\section{Measurement of Naloxone-Precipitated Withdrawal}

Somatic withdrawal was measured the day after the last escalation session at the time of the beginning of the rats' usual self-administration sessions (ie, 10-12 h into withdrawal for the LgA group and 21-23 h into withdrawal for the ShA group). Naloxone-precipitated withdrawal was scored as previously reported (Vendruscolo et al, 2011), with minor modifications, by two experimenters who were blind to group assignment. ShA and LgA rats were subcutaneously injected with $1 \mathrm{mg} / \mathrm{kg}$ naloxone and immediately placed in a $40 \mathrm{~cm}^{3}$ clear Plexiglas box. Two classes of somatic signs of opioid withdrawal were observed for $10 \mathrm{~min}$. Graded signs included wet dog shakes (2 points for 1-2 occurrences and 4 for $3+$ ) and jump attempts ( 1 point for 2-4 occurrences, 2 for 5-9, and 3 for $10+$ ). We also measured body weight loss $60 \mathrm{~min}$ after the naloxone injection (1 point per $\mathrm{g}$ lost). Checked signs included abdominal spasms (2 points), penile erection/ ejaculation/grooming of the genital area (3 points), teeth chattering ( 2 points), vocalization upon touch (3 points), swallowing movements ( 2 points), ptosis ( 2 points), abnormal posture (3 points), defecation/diarrhea ( 2 points), and profuse salivation ( 7 points). Data are expressed as the total withdrawal score.

\section{Responding for Vapor Without Sufentanil}

This control experiment was conducted with another group of drug-naive rats $(n=8)$ and was similar to the previously described self-administration experiments except that the rats were never exposed to sufentanil. Four different experimental conditions were used in $2 \mathrm{~h}$ selfadministration sessions. First, nosepokes in the active hole resulted in $10 \mathrm{~s}$ delivery of vapor without sufentanil (ie, 80/20 mixture of propylene glycol/vegetable glycerol) and initiation of a $60 \mathrm{~s}$ timeout period that was signaled by a cue light 
(vapor+cue). Second, nosepokes in the active hole resulted in $10 \mathrm{~s}$ delivery of vapor without sufentanil (vapor only); no cue light was presented. Third, nosepokes in the active hole resulted in $60 \mathrm{~s}$ cue light (cue only); no vapor was presented. Fourth, nosepokes in the active hole resulted in no consequences (no vapor/no cue). Six sessions were conducted for each experimental condition. Nosepokes in the inactive hole had no programmed consequences.

\section{Statistical Analysis}

All data are expressed as means and SEM. Data were analyzed using analysis of variance (ANOVA) with or without repeated measures (details are provided in the Results section below). When appropriate, post hoc comparisons were performed using the Duncan test. Student's unpaired $t$-tests were used for two-sample comparisons. Pearson's correlation coefficient $(r)$ was used to assess the significance of correlations. Statistical significance for all analyses was set at $p \leqslant 0.05$.

\section{RESULTS}

\section{Concentration-Response Function for Sufentanil Vapor Self-Administration}

Rats were given the opportunity to self-administer varying concentrations of sufentanil vapor in $2 \mathrm{~h}$ sessions according to the following schedule: $3.33 \mathrm{mg} / \mathrm{ml}$ for six sessions, $1.65 \mathrm{mg} / \mathrm{ml}$ for eight sessions, and $10 \mathrm{mg} / \mathrm{ml}$ for four sessions. The average number of reinforcers earned across sessions for each sufentanil concentration was calculated. The one-way repeated-measures ANOVA, with concentration as the within-subjects factor, yielded a main effect of drug concentration on active nosepokes $\left(\mathrm{F}_{2,14}=26.7\right.$, $p<0.0001)$. The number of drug deliveries was significantly higher for $1.65 \mathrm{mg} / \mathrm{ml}(p<0.01)$ and significantly lower for $10 \mathrm{mg} / \mathrm{kg}(p<0.01)$ compared with $3.33 \mathrm{mg} / \mathrm{ml}$ (Figure 1a).

There was also a significant effect of drug concentration on inactive nosepokes $\left(\mathrm{F}_{2,14}=6.6, p<0.01\right)$. The rats made fewer inactive nosepokes $(p<0.01)$ at $10 \mathrm{mg} / \mathrm{ml}$ than at the other two doses (Supplementary Table S1).

\section{Effect of Naloxone on Sufentanil Vapor Self- Administration}

The data for the number of active nosepokes for sufentanil $(10 \mathrm{mg} / \mathrm{ml})$ in $\mathrm{BL}$ sessions (ie, without naloxone injection) and in the presence of ascending concentrations of naloxone $(0.12,0.25,0.5$, and $1 \mathrm{mg} / \mathrm{kg})$ are presented in Figure $1 \mathrm{~b}$. The ANOVA, with dose and time as within-subjects factors, yielded a significant dose $\times$ time interaction $\left(F_{12,84}=10.8\right.$, $p<0.0001)$. All four naloxone doses significantly increased responding for sufentanil vapor at $30 \mathrm{~min}(p<0.0001)$ and $60 \mathrm{~min}(p<0.05$ for $0.12 \mathrm{mg} / \mathrm{kg}, p<0.01$ for $0.25 \mathrm{mg} / \mathrm{kg}$ and $0.5 \mathrm{mg} \mathrm{kg}$, and $p<0.0001$ for $1 \mathrm{mg} / \mathrm{kg}$ ) compared with BL. At $90 \mathrm{~min}$, a significant increase in responding compared with $\mathrm{BL}$ was observed for naloxone at $0.5 \mathrm{mg} / \mathrm{kg}(p<0.05)$ only. No significant effects of naloxone on responding for sufentanil were found at $120 \mathrm{~min}$.

The number of inactive nosepokes significantly (main effect of session: $F_{4,28}=4.7, p<0.01$ ) increased for naloxone at $0.125 \mathrm{mg} / \mathrm{kg}(p<0.05), 0.25 \mathrm{mg} / \mathrm{kg}(p<0.05), 0.5 \mathrm{mg} / \mathrm{kg}$
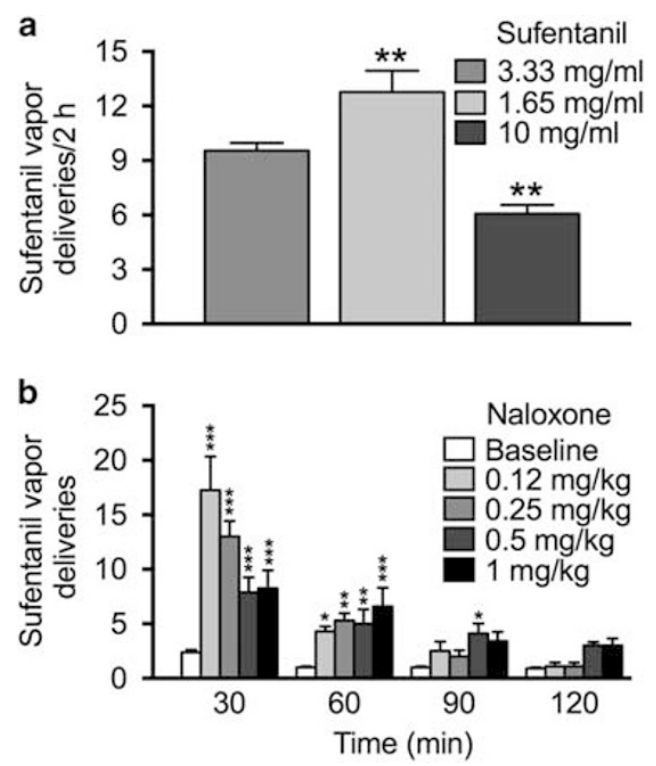

Figure I Operant sufentanil vapor self-administration. (a) Concentration-response function for sufentanil vapor self-administration. Rats $(n=8)$ self-administered sufentanil vapor ( $10 \mathrm{~s}$ delivery) in $2 \mathrm{~h}$ sessions according to the following concentration schedule (in order of presentation): $3.33 \mathrm{mg} /$ $\mathrm{ml}$ for six consecutive sessions, $1.65 \mathrm{mg} / \mathrm{ml}$ for eight consecutive sessions, and $10 \mathrm{mg} / \mathrm{ml}$ for four consecutive sessions. Bars represent the mean number of reinforcers ( + SEM) earned across sessions for each of the three different sufentanil concentrations. **p $<0.0$ I, different from $3.33 \mathrm{mg} / \mathrm{ml}$. (b) Effect of the opioid receptor inverse agonist naloxone on sufentanil vapor self-administration. The same rats $(n=8)$ that were tested in panel (a) selfadministered sufentanil vapor $(10 \mathrm{mg} / \mathrm{ml})$ in $2 \mathrm{~h}$ sessions. They were subcutaneously injected with $0.12,0.25,0.5$, and $1 \mathrm{mg} / \mathrm{kg}$ naloxone immediately prior to sufentanil self-administration (one self-administration session per concentration). The baseline data are expressed as the average of five baseline sessions that were conducted intercalated with the naloxone tests. ${ }^{*} p<0.05$, $* * * 0.01$, **** $p<0.0001$, different from baseline at the same time of the session.

$(p<0.01)$, and $1 \mathrm{mg} / \mathrm{kg}(p<0.01)$ compared with $\mathrm{BL}$ during the total $2 \mathrm{~h}$ sessions (Supplementary Table S2).

\section{Serum Sufentanil Concentrations}

To confirm that higher levels of drug vapor exposure corresponded to higher blood concentrations of sufentanil, sufentanil $(3 \mathrm{mg} / \mathrm{ml})$ was passively administered in varying numbers of evenly spaced vapor deliveries across $2 \mathrm{~h}$ (Figure 2a). Blood samples were collected $2 \mathrm{~min}$ after the final sufentanil vapor administration (three rats per dose). A positive correlation was found between the number of deliveries of sufentanil vapor and serum sufentanil concentration (in ng/ml; $r=0.997, p<0.05$; Figure 2b).

\section{Escalation and Re-Escalation of Sufentanil Vapor Self- Administration}

A separate cohort of rats was used for this experiment $(n=16)$. Rats self-administered $3 \mathrm{mg} / \mathrm{ml}$ sufentanil for either $1 \mathrm{~h}(\mathrm{ShA} ; n=8)$ or $12 \mathrm{~h}(\operatorname{LgA} ; n=8)$ per session (Figure $3 \mathrm{a})$. Separate repeated-measures ANOVAs, with session as the within-subjects factor, were conducted for ShA and LgA rats. The ANOVA yielded a main effect of session on active nosepokes in ShA rats $\left(F_{12,84}=8.5, p<0.0001\right)$. ShA rats self- 
a Non-contingent deliveries
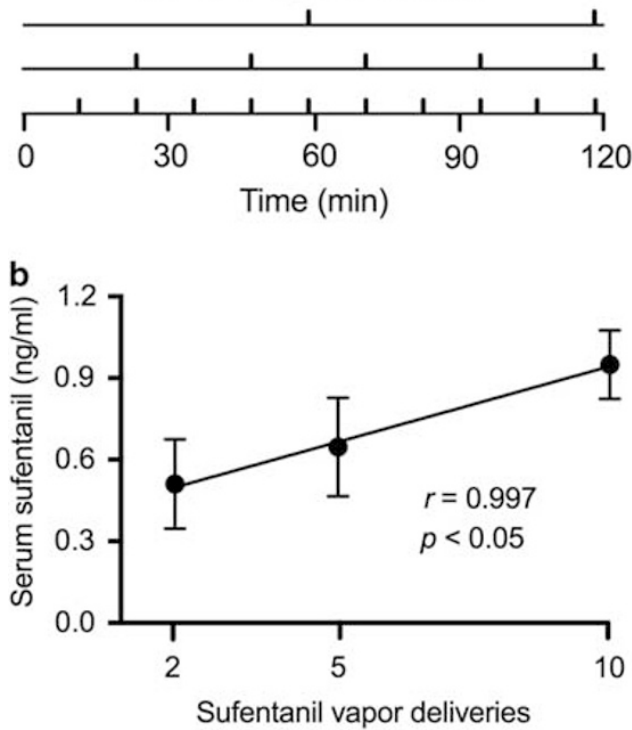

Figure 2 Serum sufentanil concentrations. (a) Timeline for non-contingent delivery of sufentanil vapor. A drug-naive cohort of rats received 2 , 5, or 10 non-contingent sufentanil vapor $(3 \mathrm{mg} / \mathrm{ml})$ deliveries ( $n=3$ rats per condition) in a $2 \mathrm{~h}$ period. The onset of vapor deliveries across the $2 \mathrm{~h}$ period was evenly spaced and timed so that the final vapor delivery occurred at minute II 8 of the session, regardless of the number of deliveries. (b) Symbols represent mean serum sufentanil concentrations ( $\mathrm{ng} / \mathrm{ml}$; +SEM) following 2,5 , and 10 sufentanil vapor deliveries. There was a significant correlation between serum sufentanil concentration and the number of sufentanil vapor deliveries $(p<0.05)$. The data are expressed as $\mathrm{ng} / \mathrm{ml}$ of serum.

administered less sufentanil vapor in session $2(p<0.01)$, session $3(p<0.05)$, and session $4(p<0.05)$ and more sufentanil vapor in session $12(p<0.05)$ and session 13 $(p<0.001)$ compared with session 1 . In LgA rats, the ANOVA yielded a main effect of session on active nosepokes $\left(F_{12,84}=5.8, p<0.0001\right)$. LgA rats self-administered more sufentanil vapor in session $10 \quad(p=0.05)$, session 11 $(p<0.05)$, session $12 \quad(p<0.0001)$, and session 13 $(p<0.0001)$ compared with session 1 .

After 6 months of forced drug abstinence, the same rats were again given the opportunity to self-administer sufentanil vapor. One rat in the ShA group died before the reescalation experiment (ShA rats, $n=7$; LgA rats, $n=8$ ). The results of the re-escalation test are shown in Figure $3 \mathrm{~b}$. The ANOVA confirmed a main effect of session in both ShA rats $\left(F_{12,72}=2.2, p<0.05\right)$ and $\operatorname{LgA}$ rats $\left(F_{12,84}=2.2, p<0.05\right)$. ShA rats self-administered more sufentanil vapor in session $12(p<0.01)$ compared with session 1. LgA rats selfadministered more sufentanil vapor in session $9(p<0.01)$, session $10(p<0.05)$, session $11(p<0.05)$, and session 13 $(p<0.05)$ compared with session 1 .

Raster plots that illustrate the number of active (up ticks) nosepokes in rats in the ShA and LgA groups during sessions 1 and 13 are shown in Figure $3 c$ and $d$. The data from the $\operatorname{LgA}$ rat shown in Figure $3 \mathrm{~d}$ exemplifies the potential use of this model for the study of drug intake after a history of drug dependence, although there was considerable variability in the re-escalation sessions among $\operatorname{LgA}$ rats. The specific LgA rat shown in Figure 3d began re-escalation at a higher level of drug self-administration compared with the end of its first escalation exposure and exhibited a further increase in intake by the end of the re-escalation period, indicating the potential of this longitudinal approach to study long-lasting drug-induced neuroadaptations in subjects with a history of drug dependence.

No significant differences were found in inactive nosepokes between the first and last sessions in ShA or LgA rats during the escalation or re-escalation tests (Supplementary Table S3).

\section{Von Frey Mechanical Sensitivity in ShA and LgA rats}

The same rats that were used for the escalation (Figure $4 \mathrm{a}$ ) and re-escalation (Figure $4 \mathrm{~b}$ ) of sufentanil vapor selfadministration were tested for mechanical sensitivity during spontaneous withdrawal. The two-way repeated-measures ANOVA yielded a group (ShA and $\operatorname{LgA}) \times$ time interaction $\left(F_{2,28}=3.7, p<0.05\right)$. The thresholds for mechanical sensitivity in $\operatorname{LgA}$ rats at T2 were significantly lower compared with their respective $\mathrm{BL}$ and compared with $\mathrm{ShA}$ rats at T2 $(p<0.01$; Figure 4a). Mechanical sensitivity thresholds did not significantly change over time in ShA rats. For the re-escalation test, LgA rats again exhibited lower mechanical sensitivity thresholds at $\mathrm{T} 2$ compared with their respective $\mathrm{BL}$ and compared with ShA rats at T2 (group $\times$ time interaction: $\mathrm{F}_{2,26}=3.8, p<0.05$; post hoc test, $p<0.01$; Figure $4 \mathrm{~b}$ ).

\section{Naloxone-Precipitated Somatic Signs of Opioid Withdrawal}

The same rats that were used for the escalation and reescalation of sufentanil vapor self-administration and mechanical sensitivity testing were also used to evaluate naloxone-precipitated withdrawal. This test was conducted after the last self-administration session (ie, session 13) during the escalation and re-escalation phases. The twotailed unpaired Student's $t$-test indicated that $\operatorname{LgA}$ rats exhibited significantly higher scores of naloxone-precipitated opioid withdrawal compared with ShA rats after escalation $\left(t_{14}=3.1, p<0.01\right.$; Figure $\left.4 c\right)$ and re-escalation $\left(t_{14}=2.9\right.$, $p<0.05$; Figure $4 \mathrm{~d}$ ) of sufentanil vapor self-administration.

\section{Responding for Vapor Without Sufentanil}

Rats were given the opportunity to nosepoke in the active hole in $2 \mathrm{~h}$ sessions to receive vapor without sufentanil accompanied by $60 \mathrm{~s}$ of cue light (vapor+cue), vapor with no cue light (vapor only), cue light without vapor (cue only), or a condition in which responding in the active hole had no consequences (no vapor/no cue). The average number of nosepokes in the active hole across six sessions for each experimental condition was calculated. The one-way repeated-measures ANOVA revealed no effect of condition on responding in the active hole, indicating that rats exhibited similar levels of responding in vapor+cue, vapor only, cue only, and no vapor/no cue conditions (Supplementary Figure S1).

\section{DISCUSSION}

We developed a new preclinical animal model of compulsive-like drug self-administration using vaporized sufentanil that was delivered using e-cigarette-type 

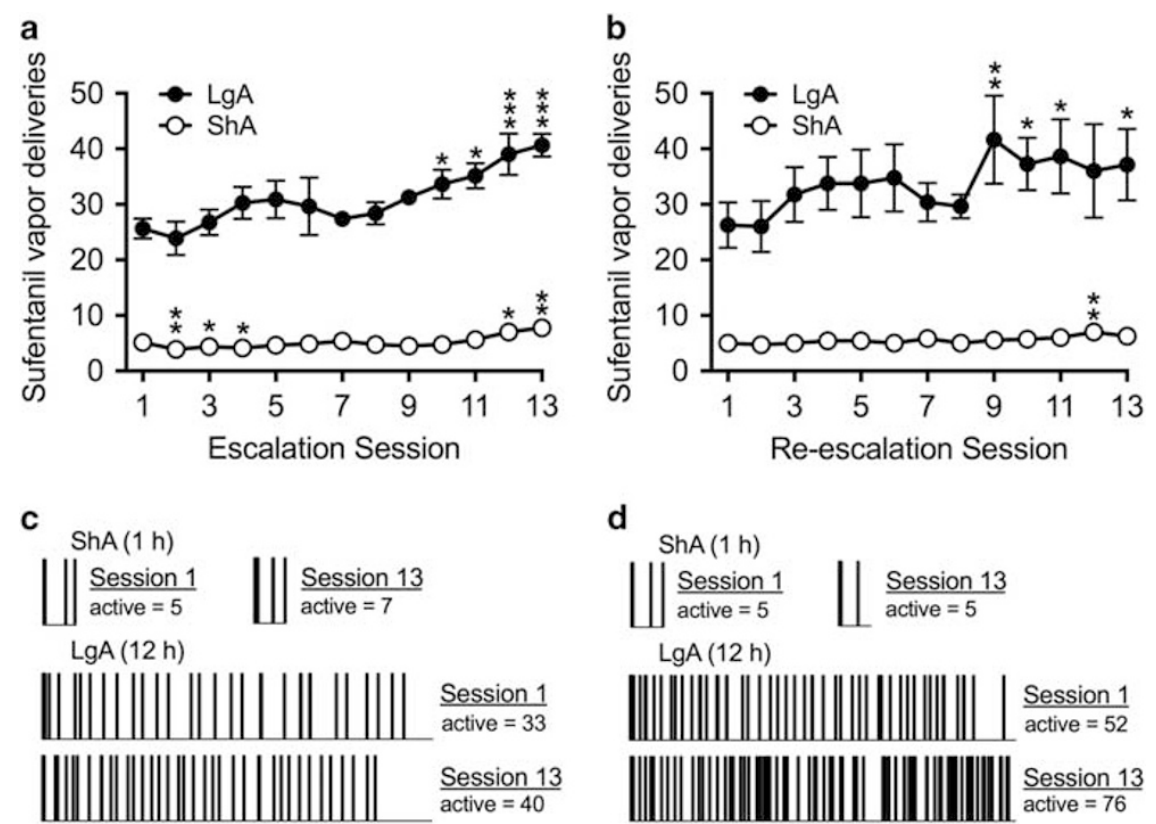

Figure 3 Escalation and re-escalation (6 months later) of sufentanil vapor self-administration. (a) Escalation of sufentanil vapor self-administration; symbols represent the mean number of sufentanil vapor deliveries ( \pm SEM) over 13 sessions. Rats that were allowed long access (LgA; $12 \mathrm{~h}$ sessions) to sufentanil vapor $(3 \mathrm{mg} / \mathrm{ml})$ exhibited dramatic escalation of drug intake over time. Rats that were allowed short access (ShA; I h sessions) to sufentanil vapor exhibited a modest decrease, then a modest increase in drug intake over time. $n=8$ per group. (b) Re-escalation of sufentanil vapor self-administration. The same ShA and $\operatorname{LgA}$ rats that were used in the escalation experiment (panel $[\mathrm{a}]$ ) were used for the re-escalation experiment 6 months later. $\operatorname{LgA}$ rats exhibited significant reescalation of drug deliveries over 13 sessions. ShA rats exhibited more stable drug intake over time. ShA rats, $n=7 ;$ LgA rats, $n=8$. * $p<0.05, * * p<0.0$ I, ***** $p<0.00$ I, compared with session I. (c, d) Raster plots that illustrate the number of active nosepokes in one representative ShA rat and one representative LgA rat during sessions I and 13 for the escalation (c) and re-escalation (d) experiments. In LgA rats, the increase in sufentanil intake was reflected by a decrease in the inter-infusion interval over the entire $12 \mathrm{~h}$ session.

technology. The rats readily self-administered sufentanil vapor, resulting in detectable serum levels of drug. Consistent with intravenous opioid self-administration studies, rats that had extended access to sufentanil (LgA rats) exhibited escalation of sufentanil vapor self-administration, mechanical hypersensitivity during spontaneous withdrawal, and an increase in somatic signs of naloxoneprecipitated withdrawal compared with ShA rats. Thus, the operant self-administration of sufentanil vapor produced many biological effects that resemble opioid addiction in humans.

\section{Concentration-Dependent Sufentanil Vapor Self- Administration}

When given the opportunity to nosepoke for a $10 \mathrm{~s}$ sufentanil vapor delivery at a concentration of $3.33 \mathrm{mg} / \mathrm{ml}$, the rats readily acquired and maintained their operant behavior to obtain an average of 9.5 reinforcers in $2 \mathrm{~h}$. Responding increased when the sufentanil concentration was decreased to $1.65 \mathrm{mg} / \mathrm{ml}$ (average of 12.8 reinforcers), and responding decreased when the concentration was increased to $10 \mathrm{mg} / \mathrm{ml}$ (average of 6 reinforcers), indicating that the rats adjusted their intake based on the drug concentration and presumably the pharmacological effects of the drug. Dose-dependent sufentanil (nebulizer) vapor self-administration has been previously reported (Jaffe et al, 1989) and is consistent with the dose-dependent pattern of drug intake that is frequently observed in intravenous opioid self-administration studies (eg, Wade et al, 2015). Satiety, motor effects, and aversive effects of the drug are hypothesized to modulate drug selfadministration in rats (for discussion, see Panlilio et al, 2003). Additionally, based on extensive data on the regulation of drug intake by inhalation in tobacco smokers (Herning et al, 1981), other factors modulate responding for sufentanil vapor, including proximity to the vapor port and the frequency and depth of breathing during drug exposure. Juarez-Portilla et al (2017) reported that mice regulated their vaporized methamphetamine intake by spending less time in a chamber with nebulized methamphetamine vapor when the drug concentration was increased. In the present study, the significant correlation between the number of sufentanil vapor deliveries and serum drug levels suggests that the levels of responding for sufentanil vapor are proportional to drug intoxication. It is important to note that the rats were passively exposed to vapor for the determination of serum sufentanil levels. Higher serum drug levels would likely be achieved in a rat that is trained to selfadminister sufentanil because of its proximity to the vapor port (see Supplementary Video 1).

An FR1 schedule of reinforcement that requires low workload to obtain the drug was used in the present study. Future studies to assess relative motivation for opioid vapor self-administration would benefit from the utilization of different schedules of reinforcement and procedures (eg, FR5, progressive ratio, extinction, punishment, reinstatement). 

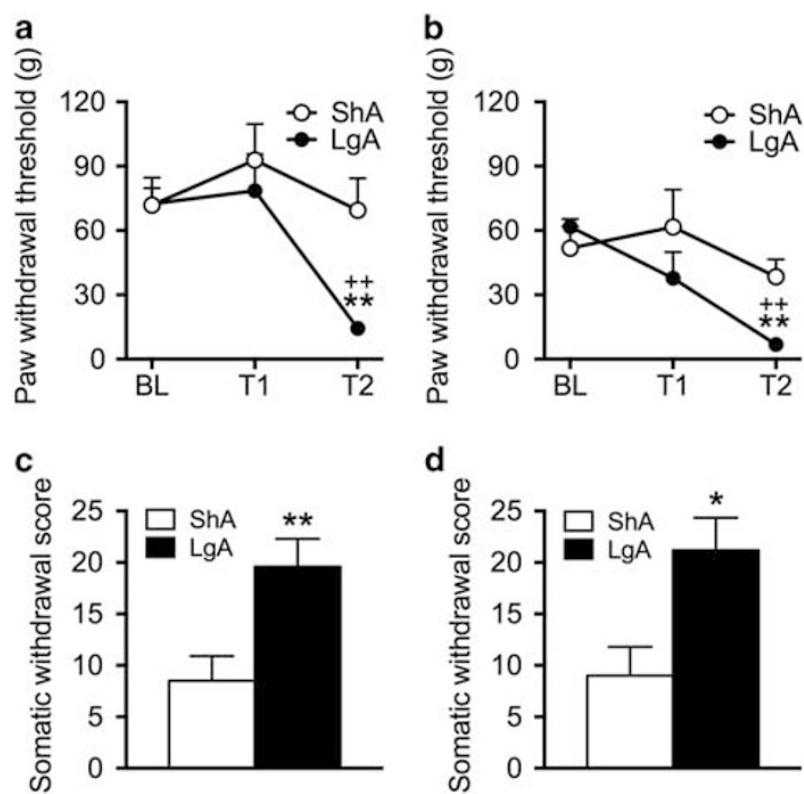

Figure 4 Mechanical sensitivity (a, b) and somatic signs of opioid withdrawal $(c, d)$ after escalation and re-escalation of sufentanil vapor selfadministration. (a) Symbols represent mechanical sensitivity measured by paw withdrawal thresholds (g; +SEM) using von Frey filaments. LgA and ShA rats (see Figure 3 for self-administration data) exhibited similar paw withdrawal thresholds at baseline (BL; conducted before any exposure to sufentanil) and test I ( $\mathrm{TI}$; conducted before the second self-administration session). LgA but not ShA rats exhibited lower paw withdrawal thresholds at T2 (conducted before the tenth self-administration session). (b) The same ShA and LgA rats were tested again for mechanical sensitivity 6 months later. Results that were identical to escalation were found (ie, no group differences for $\mathrm{BL}$ and $\mathrm{TI}$ but a significant decrease in mechanical thresholds in LgA rats at T2). $* p<0.05$, ${ }^{*} * p<0.01$, different from ShA rats; ${ }^{++} p<0.01$, different from BL. (c, d) Bars represent somatic withdrawal scores (+SEM) following naloxone-precipitated opioid withdrawal after escalation (c) and re-escalation (d). Rats were given a subcutaneous injection of naloxone $(\mathrm{l} \mathrm{mg} / \mathrm{kg}$ ) after the last self-administration session (ie, session 13) of the escalation and re-escalation experiments. LgA rats $(n=8)$ after both the escalation and the re-escalation experiments exhibited significantly higher scores of naloxone-precipitated withdrawal compared with ShA rats $(n=7)$. * $p<0.05$, *** $p<0.01$, different from ShA rats.

\section{Sufentanil Vapor Reinforcement Involves $\mu$-Opioid Receptors}

Naloxone administration prior to sufentanil vapor selfadministration significantly increased responding for sufentanil (about four times) compared with BL levels. Similar results in opioid nondependent rats intravenously selfadministering opioids (Kenny et al, 2006; Koob et al, 1984; Weeks and Collins, 1976) are frequently interpreted as an agonist-antagonist competitive interaction at $\mu$-opioid receptors, in which an increase in responding is associated with a decrease in agonist reinforcer efficacy (ie, a shift to the right of the opioid agonist dose-response function in the presence of naloxone). Also, naloxone $(1 \mathrm{mg} / \mathrm{kg}$ ) reduced responding for sufentanil in a previous study of sufentanil vapor self-administration using a nebulizer system (Jaffe et al, 1989), suggesting that the dose of naloxone was too high to allow competition by sufentanil, and so extinction or general behavior disruption likely explains the decreased responding for nebulized sufentanil in the study by Jaffe et al (1989). In aggregate, we found that different doses of naloxone similarly increased sufentanil self-administration (about 20 deliveries in $2 \mathrm{~h}$ ), indicating that the lowest dose tested of naloxone was sufficiently high to produce a maximal effect (ie, ceiling effect).

\section{Escalation and Re-Escalation of Sufentanil Vapor Self- Administration}

As previously demonstrated for intravenous opioid selfadministration, LgA rats developed tolerance and escalated their drug intake over time. In contrast, ShA rats exhibited more stable levels of responding over time, although a small but significant increase in drug intake was observed in the last two self-administration sessions in the ShA group. Escalation of sufentanil vapor self-administration in LgA rats using a nebulizer system was not detected in previous studies (Jaffe et al, 1989; Weinhold et al, 1993), possibly because the rats in these studies did not receive sufficiently high amounts of sufentanil to develop tolerance or negative emotional-like states during withdrawal, which are hypothesized to drive compulsive-like responding with extended access to drugs (Koob et al, 2014). In the present study, we observed naloxone-precipitated somatic signs of withdrawal, tactile hypersensitivity during spontaneous withdrawal, and characteristic self-injurious gnawing (data not recorded), all of which are hypothesized to drive compulsive-like drug intake via negative reinforcement mechanisms (Evans and Cahill, 2016; Koob et al, 2014), only in LgA rats.

Information about the persistence of escalated drug intake after a history of drug dependence or prior escalation in animal models is scarce in the literature. In one study, rats that received increasing doses of morphine for 4 days exhibited an increase in intravenous remifentanil selfadministration when subsequently tested $24 \mathrm{~h}$ into withdrawal (Cooper et al, 2008). In another study, rats that had a history of escalation of heroin self-administration started at escalated responding levels and further escalated their drug intake when they were again given access to heroin selfadministration after 2 weeks (Wade et al, 2017) or 1 month (Schlosburg et al, 2013) of forced abstinence. One advantage of the present vapor inhalation model is that it allows significantly longer longitudinal studies that would otherwise be difficult with intravenous catheters, requiring long-term patency. Thus, we tested the persistence of escalated drug intake after 6 months of forced abstinence. We found that sufentanil vapor self-administration returned to $\mathrm{BL}$ levels after 6 months of forced abstinence but re-escalated in a similar, even somewhat accelerated rate (9 vs 10 sessions). Also, to this point, there were individual differences in reescalation, exemplified by the individual shown in Figure $3 \mathrm{c}$ and $\mathrm{d}$, bottom trace. One possible explanation for these results is that 6 months of forced abstinence was sufficient to reverse most of the tolerance that had developed during the first escalation experiment. Further studies will be required to determine what history of sufentanil exposure will facilitate re-escalation and what duration of abstinence will protect against more rapid re-escalation.

Similarly, the present study found that a key neuroadaptation that is associated with opioid dependence (ie, mechanical hypersensitivity) also returned to $\mathrm{BL}$ levels after 6 months of abstinence. Rats that had previously received 5 days of heroin injections exhibited a significant increase in 
mechanical sensitivity when they were tested $6 \mathrm{~h}$ into withdrawal (Park et al, 2015). Célèrier et al (2001) reported that 12 daily systemic injections of low heroin doses $(0.25 \mathrm{mg} / \mathrm{kg})$ caused mechanical hyperalgesia that lasted for 7 days, whereas a similar treatment regimen with high-dose heroin $(2.5 \mathrm{mg} / \mathrm{kg})$ caused mechanical hyperalgesia that persisted for 10 days, indicating that higher opioid doses can cause longer-lasting hyperalgesia. Consistent with these reports, we found that mechanical sensitivity returned to $\mathrm{BL}$ levels after 6 months of protracted abstinence, and mechanical hypersensitivity was observed again after the reescalation of sufentanil vapor self-administration. Finally, naloxone-precipitated somatic signs of withdrawal after both escalation and re-escalation were equal in intensity, suggesting no residual sensitization of the withdrawal response.

\section{Advantages and Disadvantages of Operant Vapor Self- Administration}

There are several advantages in using the present model of operant vapor self-administration. First, it models a route of administration that is common in humans. Second, no surgery is required, resulting in less stress to the animals and no need for recovery. Third, it allows self-administration studies throughout the species' natural lifespan, including longitudinal studies with multiple periods of drug selfadministration and withdrawal. In intravenous drug selfadministration studies, catheter failure is often a critical limitation in experimental designs. Maintaining patent catheters for long periods of time, especially under conditions of extended drug access, can be challenging. Fourth, the present model can be especially useful in studies in which catheterization may be difficult or the time window for testing is narrow (eg, in adolescence or earlier stages of development). Fifth, the absence of a tether that is connected to an intravenous access port may allow simultaneous studies in which such a tether is necessary (eg, intracranial selfstimulation, calcium imaging, optogenetics, and microdialysis) while the animals self-administer drug vapor. The absence of a tether would also permit experiments in which more than one animal is tested at the same time (eg, drug intake in social or agonistic contexts). Sixth, multiple drugs can be vaporized, with no need for dual catheterization or dual infusion lines, which may be particularly useful for studies that involve the intake of multiple drugs in the same session or the choice of different drugs within the same session. Pharmacological effects of passively administered vapor using similar apparatus as the one presented herein have been reported for $\Delta^{9}$-tetrahydrocannabinol (Nguyen et al, 2016b), methamphetamine, 3,4-methylenedioxypyrovalerone, and mephedrone (4-methylmethcathinone; Nguyen et al, 2016a). Seventh, this apparatus can be used in many different species of laboratory animal subjects.

The possible disadvantages of the model that is presented herein are fewer but should be considered. First, the drug concentration may not be easily matched to body weight. Second, the precise amount of drug that is absorbed is currently difficult to determine, although blood levels can be measured for validation. These concerns will likely be clarified or alleviated as additional data are generated using these approaches.
Finally, nose-poking for rats is a prepotent response; thus the animals maintain a $\mathrm{BL}$ level of activity that includes nosepokes in the active and inactive holes. However, unlike responding in the active holes, responding in the inactive holes did not vary systematically with sufentanil dose, naloxone treatment, or escalation. Also, a control experiment indicated that responding for vapor itself (propylene glycol/ vegetable glycerol) without sufentanil with or without the presence of a cue light was not significantly different from responding without any consequences.

In summary, we developed and validated a model of compulsive-like drug self-administration via sufentanil inhalation that has face and construct validity. Our model is a promising alternative for studies of drug addiction and has many unique features and advantages, albeit with some disadvantages. This model may ultimately help elucidate the neurobiology of drug addiction, with the goal of developing safe and effective approaches for the prevention and treatment of drug use disorders.

\section{FUNDING AND DISCLOSURE}

The National Institute on Drug Abuse, Intramural Research Program supported this work. This work was also funded in part by extramural grant support from the National Institutes of Health (R01 DA035281 to MAT and a subcontract of R44 DA041967 to MAT, R01 AA022977 to OG, and R21 DA036691 to OG). MC is the proprietor of La Jolla Alcohol Research and PI of an SBIR grant (R44 DA041967) that supports the commercialization of the inhalation equipment. SAV consults for La Jolla Alcohol Research. The other authors declare no conflict of interest.

\section{ACKNOWLEDGMENTS}

We thank Michael Arends for editorial assistance and Dr Carrie Wade for helpful comments on an earlier draft of the manuscript. We also thank Shawn M Aarde for the initial development and validation of the vapor inhalation technique.

\section{REFERENCES}

Ahmed SH, Walker JR, Koob GF (2000). Persistent increase in the motivation to take heroin in rats with a history of drug escalation. Neuropsychopharmacology 22: 413-421.

Barbier E, Vendruscolo LF, Schlosburg JE, Edwards S, Juergens N, Park PE et al (2013). The NK1 receptor antagonist L822429 reduces heroin reinforcement. Neuropsychopharmacology 38: 976-984.

Célèrier E, Laulin JP, Corcuff JB, Le Moal M, Simonnet G (2001). Progressive enhancement of delayed hyperalgesia induced by repeated heroin administration: a sensitization process. J Neurosci 21: 4074-4080.

Centers for Disease Control and Prevention (CDC) (2008). Nonpharmaceutical fentanyl-related deaths-multiple states, April 2005-March 2007. MMWR Morb Mortal Wkly Rep 57: 793-796.

Chaplan SR, Bach FW, Pogrel JW, Chung JM, Yaksh TL (1994). Quantitative assessment of tactile allodynia in the rat paw. $J$ Neurosci Methods 53: 55-63.

Cooper ZD, Truong YN-T, Shi Y-G, Woods JH (2008). Morphine deprivation increases self-administration of the fast- and short- 
acting mu-opioid receptor agonist remifentanil in the rat. $J$ Pharmacol Exp Ther 326: 920-929.

Dowell D, Haegerich TM, Chou R (2016). CDC Guideline for Prescribing Opioids for Chronic Pain - United States, 2016. MMWR Recomm Rep 65: 1-49.

Edwards S, Vendruscolo LF, Schlosburg JE, Misra KK, Wee S, Park PE et al (2012). Development of mechanical hypersensitivity in rats during heroin and ethanol dependence: alleviation by $\mathrm{CRF}_{1}$ receptor antagonism. Neuropharmacology 62: 1142-1151.

Evans CJ, Cahill CM (2016). Neurobiology of opioid dependence in creating addiction vulnerability. F1000Research 5 (F1000 Faculty Rev) 1748.

Frank RG, Pollack HA (2017). Addressing the fentanyl threat to public health. N Engl J Med 376: 605-607.

Gasior M, Bond M, Malamut R (2016). Routes of abuse of prescription opioid analgesics: a review and assessment of the potential impact of abuse-deterrent formulations. Postgrad Med 128: 85-96.

Herning RI, Jones RT, Bachman J, Mines AH (1981). Puff volume increases when low-nicotine cigarettes are smoked. Br Med J Clin Res Ed 283: 187-189.

Jaffe AB, Sharpe LG, Jaffe JH (1989). Rats self-administer sufentanil in aerosol form. Psychopharmacology (Berl) 99: 289-293.

Juarez-Portilla C, Kim RD, Robotham M, Tariq M, Pitter M, LeSauter J et al (2017). Voluntary inhalation of methamphetamine: a novel strategy for studying intake non-invasively. Psychopharmacology (Berl) 234: 739-747.

Kenny PJ, Chen SA, Kitamura O, Markou A, Koob GF (2006). Conditioned withdrawal drives heroin consumption and decreases reward sensitivity. J Neurosci 26: 5894-5900.

Kintz P, Villain M, Dumestre V, Cirimele V (2005). Evidence of addiction by anesthesiologists as documented by hair analysis. Forensic Sci Int 153: 81-84.

Koob GF, Buck CL, Cohen A, Edwards S, Park PE, Schlosburg JE et al (2014). Addiction as a stress surfeit disorder. Neuropharmacology 76 Pt B: 370-382.

Koob GF, Pettit HO, Ettenberg A, Bloom FE (1984). Effects of opiate antagonists and their quaternary derivatives on heroin selfadministration in the rat. J Pharmacol Exp Ther 229: 481-486.

Lenoir M, Ahmed SH (2007). Heroin-induced reinstatement is specific to compulsive heroin use and dissociable from heroin reward and sensitization. Neuropsychopharmacology 32: 616-624.

Lenoir M, Ahmed SH (2008). Supply of a nondrug substitute reduces escalated heroin consumption. Neuropsychopharmacology 33: $2272-2282$.
Nguyen JD, Aarde SM, Cole M, Vandewater SA, Grant Y, Taffe MA (2016a). Locomotor stimulant and rewarding effects of inhaling methamphetamine, MDPV, and mephedrone via electronic cigarette-type technology. Neuropsychopharmacology 41: 2759-2771.

Nguyen JD, Aarde SM, Vandewater SA, Grant Y, Stouffer DG, Parsons LH et al (2016b). Inhaled delivery of $\Delta$ (9)-tetrahydrocannabinol (THC) to rats by e-cigarette vapor technology. Neuropharmacology 109: 112-120.

Panlilio LV, Katz JL, Pickens RW, Schindler CW (2003). Variability of drug self-administration in rats. Psychopharmacology (Berl) 167: 9-19.

Park PE, Schlosburg JE, Vendruscolo LF, Schulteis G, Edwards S, Koob GF (2015). Chronic CRF1 receptor blockade reduces heroin intake escalation and dependence-induced hyperalgesia. Addict Biol 20: 275-284.

Park PE, Vendruscolo LF, Schlosburg JE, Edwards S, Schulteis G, Koob GF (2013). Corticotropin-releasing factor (CRF) and $\alpha 2$ adrenergic receptors mediate heroin withdrawal-potentiated startle in rats. Int J Neuropsychopharmacol 16: 1867-1875.

Schlosburg JE, Vendruscolo LF, Bremer PT, Lockner JW, Wade CL, Nunes AAK et al (2013). Dynamic vaccine blocks relapse to compulsive intake of heroin. Proc Natl Acad Sci USA 110: 9036-9041.

Schmeichel BE, Barbier E, Misra KK, Contet C, Schlosburg JE, Grigoriadis D et al (2015). Hypocretin receptor 2 antagonism dose-dependently reduces escalated heroin self-administration in rats. Neuropsychopharmacology 40: 1123-1129.

Vendruscolo LF, Schlosburg JE, Misra KK, Chen SA, Greenwell TN, Koob GF (2011). Escalation patterns of varying periods of heroin access. Pharmacol Biochem Behav 98: 570-574.

Wade CL, Kallupi M, Hernandez DO, Breysse E, de Guglielmo G, Crawford E et al (2017). High-frequency stimulation of the subthalamic nucleus blocks compulsive-like re-escalation of heroin taking in rats. Neuropsychopharmacology 42: 1850-1859.

Wade CL, Vendruscolo LF, Schlosburg JE, Hernandez DO, Koob GF (2015). Compulsive-like responding for opioid analgesics in rats with extended access. Neuropsychopharmacology 40: 421-428.

Weeks JR, Collins RJ (1976). Changes in morphine selfadministration in rats induced by prostaglandin E1 and naloxone. Prostaglandins 12: 11-19.

Weinhold LL, Sharpe LG, Jaffe JH (1993). Housing conditions influence acquisition of sufentanil aerosol self-administration in rats. Pharmacol Biochem Behav 44: 141-144.

Wilkerson RG, Kim HK, Windsor TA, Mareiniss DP (2016). The opioid epidemic in the United States. Emerg Med Clin North Am 34: e1-e23.

Supplementary Information accompanies the paper on the Neuropsychopharmacology website (http://www.nature.com/npp) 